\title{
DECOMPOSITION OF POSITIVE PROJECTIONS ONTO JORDAN ALGEBRAS
}

\author{
A. GUYAN ROBERTSON
}

\begin{abstract}
If a positive projection $P$ from a $C^{*}$-algebra onto a Jordan algebra can be decomposed as a sum of maps satisfying certain inequalities of the Schwarz type, then $P$ is actually a sum of completely positive and completely copositive maps.
\end{abstract}

Suppose that $P$ is a positive unital projection on a $C^{*}$-algebra $A$ such that $P(A)_{s a}$ is a $J C$-algebra. $P$ is said to be decomposable if it is the sum of completely positive and completely copositive maps on $A$. It was shown in [6] that $P$ is decomposable if and only if $P(A)_{s a}$ is reversible in the sense that it is closed under symmetric products of the type $x_{1} x_{2} \cdots x_{n}+x_{n} \cdots x_{2} x_{1}$. Using this result, it was shown in [5] that $P$ is decomposable whenever it can be expressed as a sum of 2-positive and 2-copositive maps. We now give an improvement to the latter result, using a technique of [2].

If $A$ is a unital $C^{*}$-algebra and $H$ is a Hilbert space, then a linear map $\phi$ : $A \rightarrow B(H)$ is called a Schwarz map [4] if it satisfies the inequality $\phi(a)^{*} \phi(a) \leqslant$ $\phi\left(a^{*} a\right)$ for all $a \in A$. Similarly $\phi$ is an anti-Schwarz map if it satisfies $\phi(a) \phi(a)^{*} \leqslant$ $\phi\left(a^{*} a\right)$ for all $a \in A$. Every unital 2-positive map $\phi$ is a Schwarz map, but not conversely [1]. Similarly every unital 2-copositive map is an anti-Schwarz map. It follows from [1, Lemma 2.1] that a unital linear map $\phi$ is a Schwarz map if and only if

$$
\phi \otimes I_{2}\left(1 \otimes e_{11}+a \otimes e_{12}+a^{*} \otimes e_{21}+a^{*} a \otimes e_{22}\right) \geqslant 0,
$$

for all $a \in A$. There is obviously a similar condition for anti-Schwarz maps.

In order to study the decomposition of $P$ we need to consider nonunital maps $\phi$. The ideas of [2, Lemma 3] are useful here. Let $h=\phi(1)^{1 / 2}$, with support projection p. Then $h^{-1}$ exists as a positive (unbounded) selfadjoint operator affiliated with $p B(H) p$, and we can define a positive unital linear map $\tilde{\phi}$ on $A$ by $\tilde{\phi}(a)=$ $h^{-1} \phi(a) h^{-1}$. We shall say that $\phi$ is sesqui-positive if $\tilde{\phi}$ is a Schwarz map and that $\phi$ is sesqui-copositive if $\tilde{\phi}$ is an anti-Schwarz map. We then have the following characterization of sesqui-positive maps, which has an obvious analogue for sesquicopositive maps.

LEMMA 1. The following statements are equivalent, for a positive linear map $\phi$ : $A \rightarrow B(H)$.

Received by the editors February 5, 1985 and, in revised form, April 2, 1985. 1980 Mathematics Subject Classification. Primary 46L05; Secondary 17C65.

01986 American Mathematical Society $0002-9939 / 86 \$ 1.00+\$ .25$ per page 
(1) $\phi$ is sesqui-positive.

(2) $\phi\left(a^{*} a\right) \geqslant \phi(a)^{*} \phi(1)^{-1} \phi(a)$, for all $a \in A$.

(3) $\phi \otimes I_{2}\left(1 \otimes e_{11}+a \otimes e_{12}+a^{*} \otimes e_{21}+a^{*} a \otimes e_{22}\right) \geqslant 0$, for all $a \in A$.

Proof. The right-hand side of the inequality in (2) is to be interpreted as the strong limit of the sequence $\phi(a)^{*}(\phi(1)+1 / n)^{-1} \phi(a)$ as $n \rightarrow \infty$, as in [3]. The equivalence of the three conditions follows from [2, Lemma 3, 1, Lemma 2.1].

We need two more preliminary lemmas before proving the main result.

Lemma 2. If $\phi: A \rightarrow B(H)$ is a sesqui-positive (sesqui-copositive) linear map, then the same is true for the second dual map $\phi^{* *}$.

Proof. This follows easily from condition (3) of Lemma 1, by approximating elements of $A^{* *}$ in the $\sigma$-strong* topology.

Lemma 3. Let $M$ be a von Neumann algebra, $B$ a JW-subalgebra of $M_{s a}$ and $\phi a$ positive linear map on $M$ such that $\phi(x) \leqslant x$ whenever $0 \leqslant x \in B$. Then $\phi(b)=$ $\phi(1) b=b \phi(1)$ for all $b \in B$.

Proof. Given a projection $p \in B$, we have $0 \leqslant \phi(p) \leqslant p$, so that $(1-p) \phi(p)=$ 0 . Replacing $p$ by $1-p$ gives $p \phi(1-p)=0$, and subtraction of these two equations results in $\phi(p)=p \phi(1)$. The result follows, since $B$ is the closed linear span of its projections.

Theorem 4. Let $A$ be a unital $C^{*}$-algebra and $P$ a positive unital projection on $A$ such that $B=P(A)_{\text {sa }}$ is a JC-subalgebra of $A$. If $P=\phi+\psi$, where $\phi$ is sesquipositive and $\psi$ is sesqui-copositive, with $\phi(1), \psi(1)$ invertible, then $P$ is decomposable.

Proof. It is enough to show that the second dual map $P^{* *}$ is decomposable. We may identify $B^{* *}$ with the ultraweak closure of $B$ in the von Neumann algebra $A^{* *}$, so that $B^{* *}=P^{* *}\left(A_{s a}^{* *}\right)$ is a $J W$-subalgebra of $A$ [7, Theorem 1]. Therefore, by Lemma 2, we may suppose that $A$ is a von Neumann algebra, $B$ is a $J W$-subalgebra and $P$ is a normal projection satisfying $P(A)_{s a}=B$. To show that $P$ is decomposable, it suffices to show that $B$ is reversible [6, Corollary 7.4].

Let $h=\phi(1)^{1 / 2}$ and $k=\psi(1)^{1 / 2}$. Define $\tilde{\phi}$ and $\tilde{\psi}$ to be the positive unital linear maps associated with $\phi$ and $\psi$ respectively. Then $\tilde{\phi}$ is a Schwarz map and $\tilde{\psi}$ is an anti-Schwarz map, and

$$
P(a)=h \tilde{\phi}(a) h+k \tilde{\psi}(a) k
$$

Note that $h^{2}+k^{2}=1$, from which it follows that $h$ and $k$ commute.

Now if $b \in B$ then $P(b)=b$, so by Lemma 3, $\phi(b)=h^{2} b=b h^{2}$ and $\psi(b)=$ $k^{2} b=b k^{2}$. In particular $h b=b h$ and $k b=b k$, whenever $b \in B$, so that $\tilde{\phi}(b)=$ $\tilde{\psi}(b)=b$. Since $B$ is a Jordan algebra, we also have $\tilde{\phi}\left(b^{2}\right)=\tilde{\psi}\left(b^{2}\right)=b^{2}$.

We can now show that $B$ is reversible. We may suppose that $A$ is the von Neumann algebra generated by $B$. It follows from [4, Theorem] and its anti-Schwarz analogue that $\tilde{\phi}$ is the identity map and $\tilde{\psi}$ is an anti-automorphism of order 2 of $A$ 
which fixes the elements of $B$. Also, $h, k$ belong to the centre of $A$ and

$$
P(a)=h^{2} \tilde{\phi}(a)+k^{2} \tilde{\psi}(a) .
$$

It is clear from this that $B$ is reversible, which proves the result.

Remarks. (1) The argument above proves more than stated. In fact if $A$ is the von Neumann algebra generated by a $J W$-algebra $B$ and $P$ is a positive unital projection satisfying $P\left(A_{s a}\right)=B$ and $P=\phi+\psi$, with $\phi, \psi$ as before, then

$$
P(a)=z_{1} a+z_{2} \alpha(a) \text {, }
$$

where $z_{1}, z_{2} \geqslant 0$ are in the centre of $A, z_{1}+z_{2}=1$ and $\alpha$ is a $*$ antiautomorphism of order 2 of $A$.

(2) If $B$ is a spin factor of dimension other than 3,4 or 6 , then the corresponding projection $P$ fails to have a decomposition as in [6, Theorem 4].

The author is grateful to M. D. Choi for some helpful comments on the decomposition of positive mappings.

NOTE ADDED IN PROOF. E. Størmer has kindly drawn the author's attention to a paper of T. B. Andersen, [On multipliers and order-bounded operators in $C^{*}$-algebras, Proc. Amer. Math. Soc. 25 (1970), 896-899] which proves a $C^{*}$-algebraic version of Lemma 3.

\section{REFERENCES}

1. M. D. Choi, Some assorted inequalities for positive linear maps on $C^{*}$-algebras, J. Operator Theory 4 (1980), 271-285.

2. L. T. Gardner, Linear maps of $C^{*}$-algebras preserving the absolute value, Proc. Amer. Math. Soc. 76 (1979), 271-278

3. E. H. Lieb and M. Ruskai, Some operator inequalities of the Schwarz type, Adv. in Math. 12 (1974), 269-273.

4. T. W. Palmer, Characterizations of *-homomorphisms and expectations, Proc. Amer. Math. Soc. 46 (1974), 265-272.

5. A. G. Robertson, Positive projections on $C^{*}$-algebras and an extremal positive map, J. London Math. Soc. (2) 32 (1985), 133-140.

6. E. Størmer, Decomposition of positive projections on $C^{*}$-algebras, Math. Ann. 274 (1980), 21-41.

7. E. Effros and E. Størmer, Jordan algebras of self-adjoint operators, Trans. Amer. Math. Soc. 127 (1967), 313-316.

Department of Mathematics, University of Edinburgh, Edinburgh, Scotland EH9 3JZ 